\title{
Redistribution of resistance and sensitivity to platinum during the observation period following treatment of epithelial ovarian cancer
}

\author{
YOSHIHITO YOKOYAMA ${ }^{1}$, MASAYUKI FUTAGAMI ${ }^{1}$, JUN WATANABE $^{2}$, NAOKI SATO $^{3}$, YUKIHIRO TERADA $^{3}$, \\ FUMIHARU MIURA ${ }^{4}$, TORU SUGIYAMA ${ }^{4}$, TADAO TAKANO ${ }^{5}$, NOBUO YAEGASHI ${ }^{5}$, TAKANOBU KOJIMAHARA ${ }^{6}$, \\ HIROHISA KURACHI $^{6}$, HIROSHI NISHIYAMA $^{7}$, KEIYA FUJIMORI ${ }^{7}$, TORU TASE $^{8}$ and HIDEKI MIZUNUMA $^{1}$ \\ ${ }^{1}$ Department of Obstetrics and Gynecology, Hirosaki University Graduate School of Medicine, Hirosaki, Aomori 0368562; \\ ${ }^{2}$ Department of Pathologic Analysis, Hirosaki University Graduate School of Health Sciences, Hirosaki, Aomori 0368564; \\ ${ }^{3}$ Department of Obstetrics and Gynecology, Akita University School of Medicine, Akita, Akita 0108543; \\ ${ }^{4}$ Department of Obstetrics and Gynecology, Iwate Medical University School of Medicine, Morioka, Iwate 0208505; \\ ${ }^{5}$ Department of Obstetrics and Gynecology, Tohoku University School of Medicine, Sendai, Miyagi 9808574; \\ ${ }^{6}$ Department of Obstetrics and Gynecology, Yamagata University School of Medicine, Yamagata, Yamagata 9909585; \\ ${ }^{7}$ Department of Obstetrics and Gynecology, Fukushima Medical University, Fukushima, Fukushima 9601295; \\ ${ }^{8}$ Department of Gynecology, Miyagi Cancer Center, Natori, Miyagi 9811293, Japan
}

Received August 10, 2013; Accepted December 2, 2013

DOI: $10.3892 /$ mco.2013.223

\begin{abstract}
The standard postoperative chemotherapy for epithelial ovarian cancer is a combination therapy including platinum and taxanes. The aim this study was to investigate the degree of platinum sensitivity in patients with relapsed epithelial ovarian cancer according to the treatment-free interval (TFI) and the histological tumor type. The medical records of 405 patients diagnosed with stage III/IV ovarian cancer, including 107 patients who relapsed after attaining a clinical complete response with first-line treatment, were retrospectively reviewed. The degree of platinum sensitivity was assessed by comparing the progression-free survival (PFS) following the second-line treatment. In patients with serous/endometrioid adenocarcinoma who were treated with platinum following relapse, there were significant differences in the PFS between the following groups of patients: those who relapsed within 6 months and those who relapsed between 6 and 12 months; those who relapsed between 6 and 12 months and those who relapsed between 12 and 18 months; and those who relapsed between 12 and 18 months and those who relapsed after 18 months. By contrast, in patients with clear cell/mucinous adenocarcinoma who were treated with platinum following a relapse, there were no significant differences in the PFS between patients who
\end{abstract}

Correspondence to: Dr Yoshihito Yokoyama, Department of Obstetrics and Gynecology, Hirosaki University Graduate School of Medicine, 5 Zaifu-cho, Hirosaki, Aomori 0368562, Japan E-mail: yokoyama@cc.hirosaki-u.ac.jp

Key words: relapsed epithelial ovarian cancer, platinum sensitivity, treatment-free interval, progression-free interval, histological type relapsed within 6 months and those who relapsed between 6 and 12 months, while there were significant differences in the PFS between those who relapsed between 6 and 12 months and those who relapsed after 12 months. With regard to the patients who relapsed after 12 months, the PFS of those with clear cell/mucinous adenocarcinoma was significantly shorter compared with the PFS of those with serous/endometrioid adenocarcinoma. Therefore, we considered it justified to classify patients with clear cell/mucinous adenocarcinoma who relapsed within 12 months as platinum-resistant and those who relapsed after 12 months as platinum-sensitive.

\section{Introduction}

The standard postoperative chemotherapy for epithelial ovarian cancer is currently a combination therapy including platinum and taxanes (1). Although the treatment outcome of epithelial ovarian cancer has improved, it remains unsatisfactory in terms of long-term survival. A recent study demonstrated that bevacizumab administered in combination with paclitaxel/carboplatin (TC) prolongs survival and may be used as maintenance chemotherapy (2). Furthermore, dose-dense weekly TC was reported to be significantly superior to $\mathrm{TC}$ therapy regarding progression-free survival (PFS) and overall survival (3). The therapeutic efficacy of intraperitoneal chemotherapy was also verified in a randomized controlled study (4). A combination of molecular-targeted agents or refined regimens has improved the outcome of first-line treatment for epithelial ovarian cancer.

Epithelial ovarian cancer is highly sensitive to chemotherapy and $75 \%$ of patients achieve a clinical complete response (CCR) with first-line treatment. However, several patients relapse, develop chronic disease and ultimately succumb to ovarian 
cancer. The disease-free survival of optimal disease (advanced cancer) was reported to be 18-24 months and that of suboptimal disease 18 months (5). Furthermore, a previous study assessing optimal and suboptimal disease reported a disease-free survival of 16-17 months (5). The approximate prevalence of relapse was $10 \%$ in low-risk groups, $20 \%$ in high-risk groups for early cancer, $60-70 \%$ in optimal surgery groups and $80-85 \%$ in suboptimal surgery groups for advanced cancer. Thus, $\geq 60 \%$ of patients with ovarian cancer are candidates for second-line treatment (5) and determining the second-line therapeutic options is vital for improving the outcome.

The treatment-free interval (TFI) following the first-line treatment is currently recognized as the most significant parameter for determining the optimal regimen for the treatment of relapsed cancer. Increasing the TFI results in an improved response to platinum (6). Commonly, the treatment regimen is selected for platinum-sensitive tumors with a TFI of $\geq 6$ months and for platinum-resistant tumors with a TFI of $<6$ months.

However, whether relapsed ovarian cancer with a TFI of 6-12 months may be treated as platinum-sensitive has not been determined. Furthermore, it has not been established whether tumors may be considered drug-sensitive or -resistant according to TFI, regardless of the differences in drug sensitivity according to histological type. In the present study, the medical records of a relatively large number of patients with relapsed stage III/IV epithelial ovarian cancer were reviewed, the PFS was calculated according to the TFI and the degree of platinum sensitivity was retrospectively verified with the TFI. Furthermore, we investigated the degree of platinum sensitivity with TFI according to histological type.

\section{Materials and methods}

Study population and inclusion criteria. The study population comprised 747 patients with epithelial ovarian cancer who underwent treatment at seven institutions participating in the Tohoku Gynecologic Cancer Unit between January, 2003 and December, 2007; these were: Hirosaki University Graduate School of Medicine (Hirosaki, Japan), Akita University School of Medicine (Akita, Japan), Iwate Medical University School of Medicine (Morioka, Japan), Tohoku University School of Medicine (Sendai, Japan), Yamagata University School of Medicine (Yamagata, Japan), Fukushima Medical University (Fukushima, Japan) and the Miyagi Cancer Center (Natori, Japan). Of the 747 patients, 405 were diagnosed with stage III/IV epithelial ovarian cancer, including 156 patients with relapsed or recurrent disease. Patients in whom a complete response (CR) was maintained, those who had received neoadjuvant chemotherapy, incomplete first-line chemotherapy or radiotherapy and those with an unknown prognosis were excluded; finally, a total of 107 patients with relapsed epithelial ovarian cancer after attaining a CCR with first-line treatment were assessed. CCR was defined as the cases which became negative for the tumor marker CA125 at the end of first-line treatment, with no lesions detected on computed tomography (CT) and positron emission tomography-CT. Informed consent was obtained from the patients or their family members to collect data, following approval by the Institutional Review Boards of the involved institutions.
Table I. Patient characteristics.

\begin{tabular}{|c|c|}
\hline Variables & No. of patients \\
\hline Age, years [median (range)] & $56(26-78)$ \\
\hline \multicolumn{2}{|l|}{ Histological type } \\
\hline Serous & 101 \\
\hline Endometrioid & 18 \\
\hline Clear cell & 26 \\
\hline Mucinous & 11 \\
\hline \multicolumn{2}{|l|}{ First-line regimen } \\
\hline $\mathrm{TC}$ & 135 \\
\hline $\mathrm{DC}$ & 10 \\
\hline CPT-P & 6 \\
\hline CAP & 5 \\
\hline $\begin{array}{l}\text { No. of first-line chemotherapy cycles } \\
\text { [median (range)] }\end{array}$ & $6(1-13)$ \\
\hline \multicolumn{2}{|l|}{ Debulking surgery } \\
\hline Complete & 31 \\
\hline Optimal & 39 \\
\hline Suboptimal & 86 \\
\hline \multicolumn{2}{|l|}{ Response to first-line chemotherapy } \\
\hline Complete response & 107 \\
\hline Partial response & 26 \\
\hline Stable disease & 4 \\
\hline Progressive disease & 19 \\
\hline \multicolumn{2}{|l|}{$\begin{array}{l}\text { CR according to histological type } \\
{[\mathrm{CR} / \text { non-CR }(\%)]}\end{array}$} \\
\hline Serous & $73 / 101(72.3)$ \\
\hline Endometrioid & $12 / 18(66.7)$ \\
\hline Clear cell & $16 / 26(61.5)$ \\
\hline Mucinous & $6 / 11(54.5)$ \\
\hline \multicolumn{2}{|l|}{ Recurrence sites after CR } \\
\hline Intraabdominal & 45 \\
\hline Intrapelvic & 44 \\
\hline Distant & 18 \\
\hline \multicolumn{2}{|l|}{ Second-line regimen } \\
\hline Platinum-based & 70 \\
\hline Non-platinum-based & 37 \\
\hline
\end{tabular}

TC, paclitaxel/carboplatin; DC, docetaxel/carboplatin; CPT-P, irinotecan (CPT-11)/cisplatin; CAP, cyclophosphamide/adriamycin/ cisplatin; $\mathrm{CR}$, complete response.

Patient characteristics. The recorded patient characteristics and variables included age, histological type of ovarian cancer, debulking surgery, first-line treatment, response to first-line treatment, time to relapse, site of relapse and second-line treatment (Table I). With regard to debulking surgery, the size of the residual tumor was graded as $0,<1$ and $\geq 1 \mathrm{~cm}$ for complete, optimal and suboptimal debulking, respectively. A central pathological review was conducted to assess the histological type.

The TFI was defined as the period from the completion of the first-line treatment to the initiation of second-line treatment after confirming disease relapse on imaging. Increased CA125 
Table II. Treatment-free interval (TFI) of patients who relapsed after achieving a complete response.

\begin{tabular}{lcc}
\hline Histological type & No. of patients & Median TFI $^{\mathrm{a}}$ \\
\hline All types & 107 & 11.5 \\
Serous & 73 & 11.5 \\
Endometrioid & 12 & 16.0 \\
Clear cell & 16 & $8.0^{\mathrm{b}}$ \\
Mucinous & 6 & 11.0 \\
\hline
\end{tabular}

${ }^{\mathrm{a} I n}$ months. ${ }^{\mathrm{b}}$ Serous vs. clear cell, $\mathrm{P}<0.02$.

Table III. Progression-free survival (PFS) according to the interval from the end of first-line treatment to relapse.

\begin{tabular}{lccc}
\hline & \multicolumn{2}{c}{$\begin{array}{c}\text { Interval from the end of first-line } \\
\text { treatment to relapse (months) }\end{array}$} \\
\cline { 2 - 4 } & $<6$ & $6-12$ & $>12$ \\
Variables & 20 & 37 & 50 \\
\hline $\begin{array}{l}\text { No. of patients } \\
\text { Median PFS (months) }\end{array}$ & 3.0 & 5.5 & 13.0 \\
$\begin{array}{l}\text { Second-line treatment } \\
\text { Platinum-based }\end{array}$ & 6 & & \\
Non-platinum-based & 14 & 14 & 41 \\
\hline
\end{tabular}

Table IV. Interval from the end of first-line treatment to relapse according to histological type.

\begin{tabular}{lcrrr}
\hline & & \multicolumn{2}{c}{$\begin{array}{c}\text { Interval from the end of first-line } \\
\text { treatment to relapse (months) }\end{array}$} \\
\cline { 3 - 5 } Histology & $\begin{array}{c}\text { No. of } \\
\text { patients }\end{array}$ & $<6$ & $6-12$ & $>12$ \\
\hline Serous & 73 & $10(14 \%)$ & $27(37 \%)$ & $36(49 \%)$ \\
Endometrioid & 12 & $3(25 \%)$ & $3(25 \%)$ & $6(50 \%)$ \\
Clear cell & 16 & $5(31 \%)$ & $6(38 \%)$ & $5(31 \%)$ \\
Mucinous & 6 & $2(33 \%)$ & $1(17 \%)$ & $3(50 \%)$ \\
\hline
\end{tabular}

levels alone were not considered to reflect relapse. PFS was defined as the interval from the initiation of second-line treatment for relapsed lesions to confirmed disease progression.

Statistical analysis. The degree of platinum sensitivity was calculated by comparing the PFS values. PFS was estimated using the Kaplan-Meier method and compared using the log-rank test. Hazard ratios (HRs) with $95 \%$ confidence intervals (CIs) were calculated with the Cox proportional hazards regression model. $\mathrm{P} \leq 0.05$ was considered to indicate a statistically significant difference.

\section{Results}

Patient characteristics. The patient characteristics are summarized in Table I. The median age of the patients was 56 years.
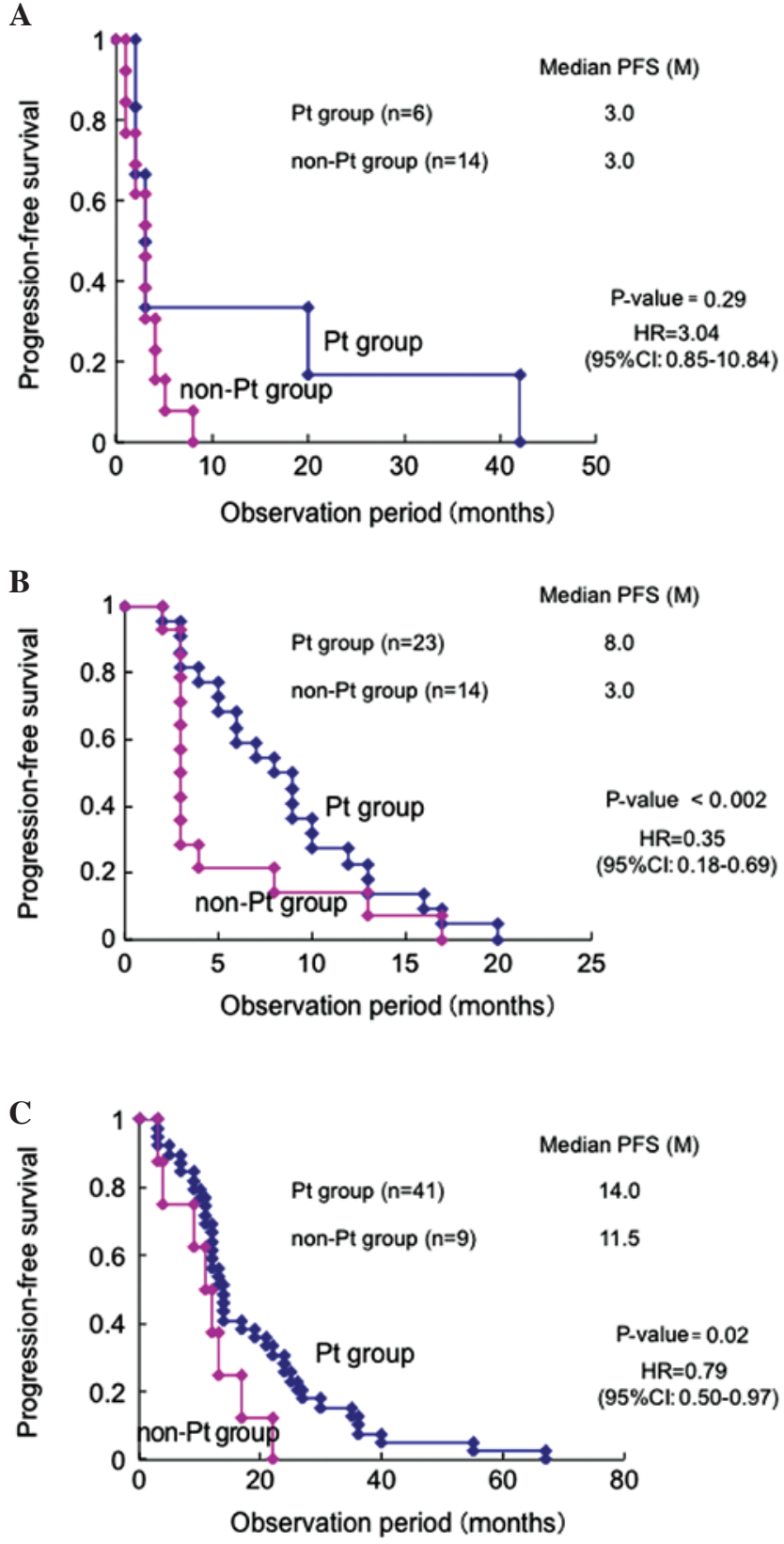

Figure 1. Comparison of progression-free survival (PFS) between the platinum $(\mathrm{Pt})$ and non-Pt groups during the observation period of all histological types. Patients who relapsed (A) $<6$ months, (B) 6-12 months and (C) $\geq 12$ months after the first-line treatment.

With regard to histological type, serous adenocarcinoma was diagnosed in 101 patients $(65 \%)$ and clear cell adenocarcinoma, which was reported to have a measurable incidence in the USA and Europe (7), was identified in 26 patients (17\%). The median number of first-line treatment cycles was 6 and the TC regimen was administered to 135 patients $(87 \%)$, whereas docetaxel/carboplatin was selected for patients with paclitaxel hypersensitivity or peripheral neurotoxicity. As regards debulking surgery, complete or optimal outcomes were achieved in 70 patients (45\%). A CR following administration of the first-line treatment was observed in 107 patients (69\%). Although the prevalence of CR following administration of the first-line regimen varied by histological type from $72.3 \%$ in serous adenocarcinoma to $54.5 \%$ in mucinous 
Table V. Progression-free survival (PFS) in patients with serous or endometrioid carcinoma and in those with clear cell or mucinous carcinoma treated with a platinum $(\mathrm{Pt})$-based regimen according to interval from the end of first-line treatment to relapse.

A, Serous/endometrioid group

\begin{tabular}{|c|c|c|c|c|}
\hline $\begin{array}{l}\text { Interval to relapse } \\
\text { after CCR } \\
\text { (months) }\end{array}$ & $\begin{array}{l}\text { No. of patients } \\
(\mathrm{n}=55) \text { with } \mathrm{Pt}- \\
\text { based regimen }\end{array}$ & & $\begin{array}{c}\text { PFS } \\
\text { (months) }\end{array}$ & \\
\hline$<6$ & 4 & & 4.5 & \\
\hline $6-12$ & 17 & & 9.0 & $\mathrm{P}=0.01 ; \mathrm{HR}=0.14 ; 95 \% \mathrm{CI}: 0.03-0.63$ \\
\hline $12-18$ & 9 & & 12.5 & $\mathrm{P}<0.05 ; \mathrm{HR}=0.56 ; 95 \% \mathrm{CI}: 0.25-0.93$ \\
\hline $18-24$ & 9 & $\mathrm{P}<0.05 ; \mathrm{HR}=0.49 ; 95 \% \mathrm{CI}: 0.22-0.96$ & 17.0 & $\mathrm{P}=0.49 ; \mathrm{HR}=1.43 ; 95 \% \mathrm{CI}: 0.51-4.08$ \\
\hline$>24$ & 16 & & 19.0 & $\mathrm{P}=0.51 ; \mathrm{HR}=0.35 ; 95 \% \mathrm{CI}: 0.54-3.34$ \\
\hline
\end{tabular}

$\mathrm{B}$, Clear cell/mucinous group

\begin{tabular}{|c|c|c|c|}
\hline $\begin{array}{l}\text { Interval to relapse } \\
\text { after CCR } \\
\text { (months) }\end{array}$ & $\begin{array}{l}\text { No. of patients } \\
(\mathrm{n}=55) \text { with } \mathrm{Pt}- \\
\text { based regimen }\end{array}$ & $\begin{array}{c}\text { PFS } \\
\text { (months) }\end{array}$ & \\
\hline$<6$ & 2 & 2.5 & \\
\hline $6-12$ & 6 & 5.5 & $\mathrm{P}=0.26 ; \mathrm{HR}, 3.67 ; 95 \% \mathrm{CI}: 0.38-9.34$ \\
\hline$>12$ & 7 & 11.0 & $\mathrm{P}<0.05 ; \mathrm{HR}, 0.33 ; 95 \%$ CI: $0.10-0.97$ \\
\hline
\end{tabular}

CCR, clinical complete response; HR, hazard ratio; CI, confidence interval.

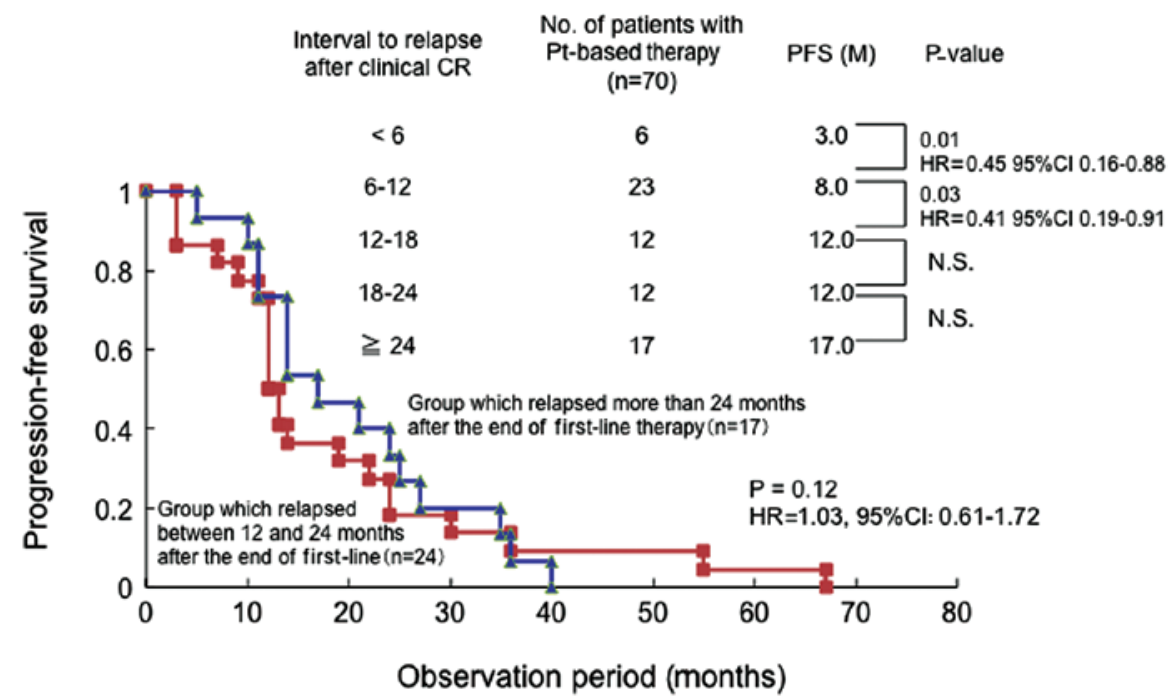

Figure 2. Comparison of the progression-free survival (PFS) by observation period in the platinum (Pt) group alone in all histological types. Patients who relapsed after 12 months were further classified every 6 months. There were no significant differences in the PFS between patients who relapsed between 12 and 24 months and those who relapsed after 24 months. N.S., not significant.

adenocarcinoma, there were no statistically significant differences in the prevalence of CR among the four histological types. The number of relapse sites was similar for abdominal and pelvic cavities. The majority of distant metastases were located in the lungs and in the mediastinal, supraclavicular or inguinal lymph nodes. In patients with a relapse following a CR (70 patients in the platinum and 37 in the non-platinum group), a second-line regimen was initiated. 
TFI in relapsed patients following CR. The median TFI in relapsed patients was 11.5 months (Table II). Significant differences in the TFI by histological type were observed between cases with serous adenocarcinoma and those with clear cell adenocarcinoma (HR=0.53; 95\% CI: 0.30-0.95; $\mathrm{P}<0.02$ ) (Table II).

PFS following second-line treatment. A total of 20,37 and 50 patients relapsed after a $\mathrm{CR}$ at $<6,6-12$ and $\geq 12$ months, respectively, and the median PFS following administration of a second-line regimen was 3.0, 5.5 and 13.0 months, respectively (Table III). There were no statistically significant differences in the distribution of the interval from the end of first-line treatment to relapse according to the histological type (Table IV). There were no significant differences in the median PFS (3.0 months) between the platinum- and non-platinum-based treatment groups who relapsed within 6 months after CR, when the PFS was compared based on the observation period for all histological types (Fig. 1A). In patients who relapsed between 6 and 12 months, the median PFS was 8.0 and 3.0 months in the platinum- and non-platinum based groups, respectively, with PFS being significantly longer in the platinum group $(\mathrm{HR}=0.35$; 95\% CI: 0.18-0.69; P<0.002) (Fig. 1B). In patients who relapsed after 12 months, the median PFS was 14.0 and 11.5 months in the platinum- and non-platinum-based groups, respectively, with the PFS being significantly longer in the platinum group (HR=0.79; 95\% CI: 0.50-0.97; P=0.02) (Fig. 1C). Therefore, the platinum group alone was further investigated. Patients who relapsed after 12 months were further classified every 6 months (Fig. 2). There were significant differences in PFS between patients who relapsed within 6 months and those who relapsed between 6 and 12 months (HR=0.45; 95\% CI: $0.16-0.88$; $\mathrm{P}=0.01$ ), as well as between those who relapsed between 6 and 12 months and those who relapsed between 12 and 18 months (HR=0.41; 95\% CI: 0.19-0.91; P=0.03; Fig. 2). However, there were no significant differences in the PFS between patients who relapsed between 12 and 18 months and those who relapsed between 18 and 24 months, or between those who relapsed between 18 and 24 months and those who relapsed after 24 months (Fig. 2). The patients who relapsed were divided into those who relapsed between 12 and 24 months and those who relapsed after 24 months and the PFS was compared between the groups: no significant differences in PFS were observed between the two groups (HR=1.03; 95\% CI: $0.61-1.72 ; \mathrm{P}=0.12$ ).

PFS by histological type following second-line treatment. The differences in PFS by histological type were investigated. The PFS in the serous/endometrioid group treated with platinum after relapse was 4.5, 9.0, 12.5, 17.0 and 19.0 months in patients who relapsed at $<6,6-12,12-18,18-24$ and $\geq 24$ months, respectively (Table V). There were significant differences in the PFS between patients who relapsed within 6 months and those who relapsed between 6 and 12 months $(\mathrm{HR}=0.14$; 95\% CI: 0.03-0.63; $\mathrm{P}=0.01$ ), as well as between those who relapsed between 6 and 12 months and those who relapsed between 12 and 18 months (HR=0.56; 95\% CI: 0.25-0.93; $\mathrm{P}<0.05)($ Table V). Although there were no significant differences in the PFS between those who relapsed between 12 and 18 and those who relapsed between 18 and 24 months, or between those who relapsed between 18 and 24 and those
A

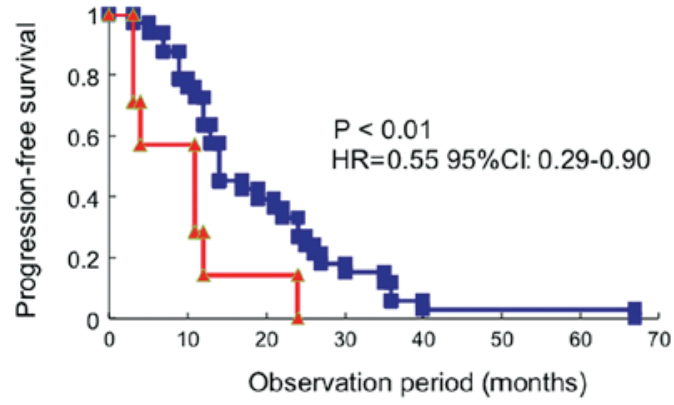

B

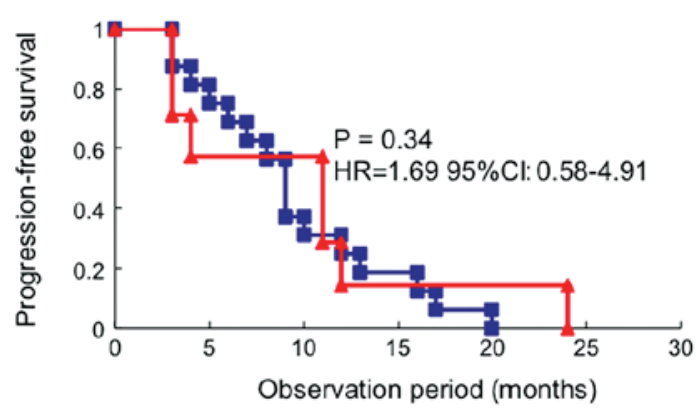

C

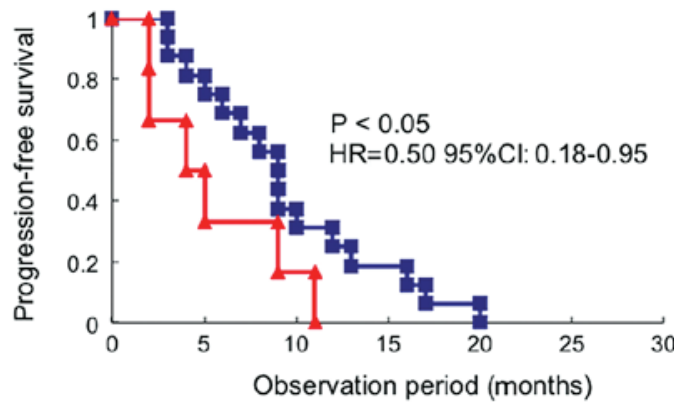

Figure 3. Evaluation of progression-free survival (PFS) of patients and redistribution of sensitivity to platinum according to the histological type. (A) Evaluation of PFS of patients with clear cell/mucinous adenocarcinoma who relapsed after 12 months $(n=7$, red line). The PFS was significantly shorter compared to that of patients with serous/endometrioid adenocarcinoma who relapsed after 12 months $(n=34$, blue line). (B) The PFS of patients with clear cell/mucinous adenocarcinoma who relapsed after 12 months $(n=7$, red line) was similar to that of patients with serous/endometrioid adenocarcinoma who relapsed between 6 and 12 months ( $n=17$, blue line). (C) Comparison of PFS between patients with clear cell/mucinous adenocarcinoma $(n=6$, red line) and those with serous/endometrioid adenocarcinoma $(n=17$, blue line) who relapsed between 6 and 12 months.

who relapsed after 24 months, there were significant differences in the PFS between patients who relapsed between 12 and 18 and those who relapsed after 18 months $(H R=0.49$; 95\% CI: 0.22-0.96; P<0.05) (Table V). Furthermore, in the clear cell/mucinous adenocarcinoma group treated with platinum as a second-line regimen, there were no significant differences in the PFS between patients who relapsed within 6 months and those who relapsed between 6 and 12 months, while there were significant differences in the PFS between patients who relapsed between 6 and 12 months and those who relapsed after 12 months ( $\mathrm{HR}=0.33 ; 95 \%$ CI: $0.10-0.97 ; \mathrm{P}<0.05$ ) (Table V). In patients who relapsed after 12 months, the PFS in the clear cell/mucinous adenocarcinoma group was significantly shorter compared to that in the serous/endometrioid 
$\mathbf{A}$

Serous/Endometrioid

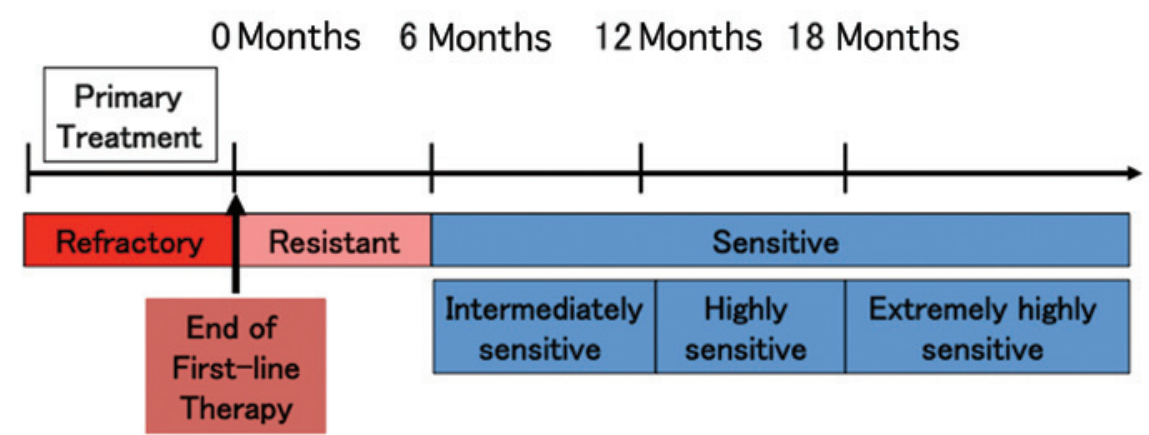

B

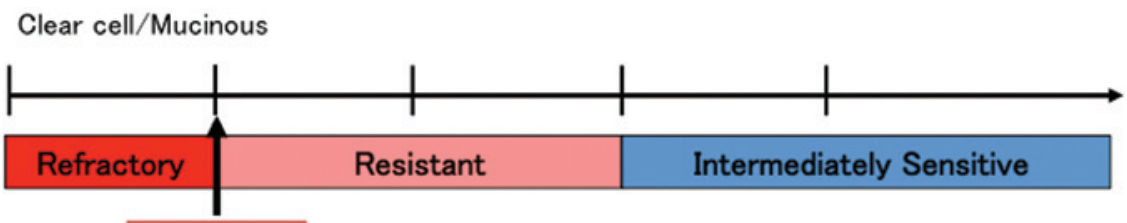

End of

First-line

Therapy

Figure 4. Redistribution of sensitivity to platinum according to the histological type. (A) Serous/endometrioid adenocarcinomas: Patients who relapsed within 6 months were considered to be 'platinum-resistant', those who relapsed between 6 and 12 months were 'intermediately sensitive', those who relapsed between 12 and 18 months were 'highly sensitive' and those who relapsed after 18 months were 'extremely highly sensitive'. (B) Clear cell/mucinous adenocarcinomas: Patients who relapsed within 12 months were considered to be 'platinum-resistant' and those who relapsed after 12 months were 'intermediately sensitive'.

adenocarcinoma group (HR=0.55; 95\% CI: 0.29-0.90; $\mathrm{P}<0.01$; Fig. 3A), but similar to that of patients who relapsed between 6 and 12 months in the serous/endometrioid adenocarcinoma group $(\mathrm{HR}=1.69 ; 95 \% \mathrm{CI}: 0.58-4.91 ; \mathrm{P}=0.34$; Fig. 3B). Furthermore, in patients who relapsed between 6 and 12 months, the PFS of the clear cell/mucinous adenocarcinoma group was significantly shorter compared to that in the serous/endometrioid adenocarcinoma group ( $\mathrm{HR}=0.50$; 95\% CI: 0.18-0.95; P<0.05; Fig. 3C).

\section{Discussion}

The aim of first-line treatment is to cure, whereas the primary goal of second-line treatment is palliation. Accordingly, less toxic and more convenient regimens should be selected, focusing on the balance between toxicity and effectiveness. Quality of life, including improving symptoms and preventing the onset of new symptoms is also preferentially maintained. Therefore, it is crucial to appropriately determine how the sensitivity or resistance to platinum is defined by TFI when selecting a regimen and TFI should also be individualized according to histological type.

Although the TFI of patients who relapsed within 6 months was suggested to indicate platinum sensitivity in this study, the sensitivity to platinum was similar between patients who relapsed between 12 and 24 months and those who relapsed after 24 months for all histological types. However, these results were inconsistent with previous findings reporting a significantly higher response rate in patients with a TFI of $\geq 24$ months $(6,8)$. In this study, the overall proportion of clear cell and mucinous adenocarcinomas, which are relatively refractory to treatment, accounted for $\sim 20 \%$ of the cases of ovarian cancer and platinum sensitivity was evaluated according to histological type. In patients with serous/endometrioid adenocarcinoma, the PFS following platinum administration exhibited a significant increase in a stepwise manner depending on the TFI (Table V). These findings suggested a novel distribution of platinum sensitivity classification. The patients who relapsed between 6 and 12 months following the completion of first-line treatment (TFI of 6-12 months) may be classified as 'intermediately sensitive', those with a TFI of 12-18 months as 'highly sensitive' and those with a TFI of $\geq 18$ months as 'extremely highly sensitive', whereas the patients with a TFI of $<6$ months were considered as platinum-resistant (Fig. 4A).

A significant difference in the PFS was only detected between patients with a TFI of 6-12 and those with a TFI of $\geq 12$ months in the clear cell/mucinous adenocarcinoma group. There were no significant differences in the PFS between patients with a TFI of $<6$ months and those with a TFI of 6-12 months. However, the PFS of patients who relapsed after 12 months was significantly shorter in the clear cell/mucinous adenocarcinoma group compared to that in the serous/endometrioid adenocarcinoma group and was similar to the PFS of patients who relapsed between 6 and 12 months in the serous/endometrioid adenocarcinoma group. Furthermore, the PFS of patients who relapsed between 6 and 12 months was significantly shorter in the clear cell/mucinous adenocarcinoma group compared to that in the serous/endometrioid adenocarcinoma group. Therefore, it may be rational to classify patients with clear cell/mucinous adenocarcinoma who relapsed within 12 months as 'platinum-resistant' and those who relapsed after 12 months as 'intermediately sensitive' (Fig. 4B).

In the 1990s, several studies focused on the identification and differentiation of platinum-sensitive from platinum-resistant relapse $(6,8,9)$. Harries and Gore (10) suggested that 
sensitivity and resistance to platinum were separated by a PFS of 6 months. Furthermore, previous studies defined sensitivity and resistance to platinum according to the clinical response, such as the frequency of CR for agents administered to relapsed patients $(6,8,9)$. However, in the present study, drug sensitivity was evaluated by the interval to disease progression (observation period), as well as the PFS of relapsed patients under platinum- and non-platinum-based treatment. It is widely accepted that the patients with a longer time interval between the completion of first-line treatment and the initiation of second-line treatment exhibit a higher response rate to the second-line regimen. In order to avoid ineffective treatment with resistant regimens, the length of the observation period should be considered on an individual basis. The results of the present study suggested that a TFI of $<6$ and $\geq 6$ months after the completion of first-line treatment may be appropriate for patients with serous/endometrioid adenocarcinoma to determine sensitivity or resistance to platinum as second-line treatment, whereas a TFI of 6 months is too short to apply to patients with clear cell/mucinous adenocarcinoma.

Thus far, an observation period of $\geq 6$ months following the end of first-line treatment has been defined as platinum-sensitive and phase III studies for patients with platinum-sensitive recurrent ovarian cancer have been designed. Although the incidence of relapsed patients with a TFI of $\geq 12$ months was reported to be $60-70 \%$ in several randomized controlled studies (11-14), the proportion of patients who relapsed between 6 and 12 months was 30-40\%, including those with platinum-resistant clear cell/mucinous adenocarcinoma. Furthermore, in the present study, although the TFI was $\geq 12$ months in the serous/endometrioid adenocarcinoma group, there were significant differences in platinum sensitivity between patients who relapsed between 12 and 18 months and those who relapsed after 18 months. Thus, future clinical studies on the selection of chemotherapy for recurrent ovarian cancer must consider the histological type and platinum sensitivity with TFI.

With regard to taxane sensitivity in recurrent ovarian cancer, it was demonstrated that the number of intervention therapies following relapse, rather than the taxane-free interval, is associated with taxane sensitivity (15). Although there were no differences in the effects of taxanes on recurrent ovarian cancer, regardless of whether the interval between the first and subsequent use of taxanes was $\leq 12$ or $\geq 24$ months, additional intervention therapies resulted in a decreased response to taxanes (15). Similarly, it was previously reported that the taxane-free interval does not affect sensitivity to taxanes (16). Therefore, drug sensitivity to taxanes and platinum must be considered separately.

Novel biological therapies, including anti-angiogenic agents, signaling inhibitors, anti-CA125 antibody and dendritic cell immunotherapy, have been developed for the treatment of recurrent ovarian cancer. Bevacizumab was reported to prolong survival in subjects with recurrent ovarian cancer (17). Molecular-targeted agents are expected to exert additive effects and platinum sensitivity is considered to play a critical role in improving prognosis. Furthermore, maintenance therapy with biological agents may eventually alter the pattern of recurrence and novel characterizations of platinum sensitivity/resistance may emerge. Although TFI is a continuous variable with a wide boundary, it is crucial to determine a definitive criterion of the sensitivity and resistance to platinum in types of ovarian cancer with a prevalence of relapse of $\geq 60 \%$, in order to enable the selection of the most efficient second-line regimen and design high-quality clinical studies.

\section{References}

1. Ozols RF, Bundy BN, Greer BE, et al; Gynecologic Oncology Group: Phase III trial of carboplatin and paclitaxel compared with cisplatin and paclitaxel in patients with optimally resected stage III ovarian cancer: a Gynecologic Oncology Group study. J Clin Oncol 21: 3194-3200, 2003.

2. Burger RA, Brady MF, Bookman MA, et al: Incorporation of bevacizumab in the primary treatment of ovarian cancer. N Engl J Med 365: 2473-2483, 2011.

3. Katsumata N, Yasuda M, Takahashi F, et al: Dose-dense paclitaxel once a week in combination with carboplatin every 3 weeks for advanced ovarian cancer: a phase 3, open-label, randomised controlled trial. Lancet 374: 1331-1338, 2009.

4. Armstrong DK, Bundy B, Wenzel L, et al: Intraperitoneal cisplatin and paclitaxel in ovarian cancer. N Engl J Med 354: 34-43, 2006.

5. Sugiyama T: Second-line treatment using novel chemotherapeutic and biologic agents. Cancer and Chemotherapy 36: 730-735, 2009 (In Japanese).

6. Markman M, Reichman B, Hakes T, et al: Responses to second-line cisplatin-based intraperitoneal therapy in ovarian cancer: influence of a prior response to intravenous cisplatin. J Clin Oncol 9: 1801-1805, 1991.

7. Hoskins PJ, Le N, Gilks B, et al: Low-stage ovarian clear cell carcinoma: population-based outcomes in British Columbia, Canada, with evidence for a survival benefit as a result of irradiation. J Clin Oncol 30: 1656-1662, 2012.

8. Gore ME, Fryatt I, Wiltshaw E, et al: Treatment of relapsed carcinoma of the ovary with cisplatin or carboplatin following initial treatment with these compounds. Gynecol Oncol 36: 207-211, 1990

9. Blackledge G, Lawton F, Redman C, et al: Response of patients in phase II studies of chemotherapy in ovarian cancer: implications for patient treatment and the design of phase II trials. Br J Cancer 59: 650-653, 1989.

10. Harries M and Gore M: Part II: chemotherapy for epithelial ovarian cancer-treatment of recurrent disease. Lancet Oncol 3: $537-545,2002$.

11. Parmar MK, Ledermann JA, Colombo N, et al: Paclitaxel plus platinum-based chemotherapy versus conventional platinum-based chemotherapy in women with relapsed ovarian cancer: the ICON4/AGO-OVAR-2.2 trial. Lancet 361: 2099-2106, 2003.

12. Pfisterer J, Plante M, Vergote I, et al: Gemcitabine plus carboplatin compared with carboplatin in patients with platinum-sensitive recurrent ovarian cancer: an intergroup trial of the AGO-OVAR, the NCIC CTG, and the EORTC GCG. J Clin Oncol 24: 4699-4707, 2006.

13. Pujade-Lauraine E, Wagner U, Aavall-Lundqvist E, et al: Pegylated liposomal doxorubicin and carboplatin compared with paclitaxel and carboplatin for patients with platinum-sensitive ovarian cancer in late relapse. J Clin Oncol 28: 3323-3329, 2010.

14. Aghajanian C, Blank SV, Goff BA, et al: OCEANS: a randomized, double-blind, placebo-controlled phase III trial of chemotherapy with or without bevacizumab in patients with platinum-sensitive recurrent epithelial ovarian, primary peritoneal, or fallopian tube cancer. J Clin Oncol 30: 2039-2045, 2012.

15. McCourt C, Dessie S, Bradley AM, et al: Is there a taxane-free interval that predicts response to taxanes as a later-line treatment of recurrent ovarian or primary peritoneal cancer? Int J Gynecol Cancer 19: 343-347, 2009.

16. Rose PG, Blessing JA, Ball HG, et al: A phase II study of docetaxel in paclitaxel-resistant ovarian and peritoneal carcinoma: a Gynecologic Oncology Group study. Gynecol Oncol 88: 130-135, 2003.

17. Aghajanian C, Blank SV, Goff BA, et al: OCEANS: a randomized, double-blind, placebo-controlled phase III trial of chemotherapy with or without bevacizumab in patients with platinum-sensitive recurrent epithelial ovarian, primary peritoneal, or fallopian tube cancer. J Clin Oncol 30: 2039-2045, 2012. 